Check for updates

Cite this: RSC Adv., 2017, 7, 46201

Received 30th June 2017

Accepted 11th September 2017

DOI: $10.1039 / c 7 r a 07259 g$

rsc.li/rsc-advances

\section{Use of a passivation layer to improve thermal stability and quality of a phosphorene/AZO heterojunction diode}

\author{
Sushil Kumar Pandey, ${ }^{a}$ Nezhueyotl Izquierdo, ${ }^{a}$ Rick Liptak ${ }^{b}$ \\ and Stephen A. Campbell (D)*a
}

\begin{abstract}
Herein, we report the effect of passivation layer composition on thermal stability as measured by Raman spectra of a phosphorene/Al-doped $\mathrm{ZnO}$ (AZO) heterostructure. Few-layer phosphorene was formed using micromechanical exfoliation on an AZO film/Si substrate. Samples were passivated by $\mathrm{Al}_{2} \mathrm{O}_{3}, \mathrm{Si}_{3} \mathrm{~N}_{4}$, or an $\mathrm{Al}_{2} \mathrm{O}_{3} / \mathrm{Si}_{3} \mathrm{~N}_{4}$ stack. Both films were deposited by atomic layer deposition. When these samples were annealed at temperatures in the range from 100 to $550{ }^{\circ} \mathrm{C}$, the nitride-only structure was found to have highest thermal sustainability, demonstrating phonon peaks of out-of-plane $\left(A_{g}{ }^{1}\right)$ and in-plane $\left(B_{2 g}\right.$ and $\mathrm{A}_{\mathrm{g}}{ }^{2}$ ) modes up to the highest temperature tested $\left(550^{\circ} \mathrm{C}\right)$. This was $100{ }^{\circ} \mathrm{C}$ higher than that for the samples with the conventionally used $\mathrm{Al}_{2} \mathrm{O}_{3}$ coating. An oxide/nitride stack permitted anneals up to $500{ }^{\circ} \mathrm{C}$. For anneals up to $200{ }^{\circ} \mathrm{C}$, the nitride-only passivated phosphorene film was found to have a significant red shift in all the three peaks. This is attributed to the effects of tensile strain. At annealing temperatures higher than $200{ }^{\circ} \mathrm{C}$, the spectra blue shifted, attaining values close to the bulk peak position; this suggested strain relaxation in the film at higher temperatures. This relaxation was also evident from a reduction in the full-width at half maximum of the Raman phonon peaks at higher annealing temperatures. Further study suggests that this shift may be due to a reduction in the stress of the passivation layer. The improvement in thermal stability is believed to be due to silicon nitride acting as a diffusion barrier, blocking phosphorus out-diffusion. To demonstrate the utility of higher temperature annealing, a phosphorene/AZO heterojunction diode was fabricated with a $\mathrm{Si}_{3} \mathrm{~N}_{4}$ passivation layer. The passivated device shows a significant improvement of the diode ideality factor with an increased annealing temperature. The improvement of the diode ideality factor is due to the significant reduction of defect concentration at high annealing temperatures. These findings will promote the development of high-performance phosphorene-based heterojunction devices.
\end{abstract}

\section{Introduction}

Atomically thin two-dimensional (2D) materials, such as graphene and transition metal dichalcogenides (TMDs), have made a significant impact in the field of electronic and optoelectronic devices. ${ }^{1-6}$ Graphene, however, has no band gap; this creates hurdles for its many device applications. ${ }^{7}$ Similarly, the modest carrier mobility of TMD materials makes them less suitable for high performance electronic and optoelectronic device applications. ${ }^{2,8,9}$ Phosphorene has attracted significant interest owing to its unique anisotropic manner, layerdependent direct band gaps, high carrier mobility, and quasione dimensional (1D) excitonic nature that are not present in the abovementioned 2D materials. ${ }^{10-18}$ Phosphorene has a band

${ }^{a}$ Department of Electrical and Computer Engineering, University of Minnesota, 200 Union Street, Minneapolis, Minnesota 55455, USA.E-mail: campb001@umn.edu

${ }^{b}$ Physics and Optical Engineering, Rose-Hulman Institute of Technology, 5500 Wabash Ave, Terre Haute, IN 47803, USA gap of $\sim 0.3 \mathrm{eV}$ in the bulk form, which increases with the reducing layer thickness, approaching $\sim 2 \mathrm{eV}$ for the monolayer. ${ }^{10-18} \mathrm{As}$ a result, there have been numerous reports on FETs and inverters fabricated in the material system. Phosphorene is also becoming an interesting material in the fabrication of solar cells and photodetectors. Theoretical studies predict that phosphorene bilayer-based solar cells have the capacity to produce an external quantum efficiency (EQE) of up to $18 \%$ in a flexible format. ${ }^{14}$ Phosphorene heterojunction $\mathrm{p}-\mathrm{n}$ diodes, although embryonic, have also been reported. ${ }^{18-20}$ These heterojunctions are generally made with other $2 \mathrm{D}$ materials (i.e. TMDs) and thus use van der Waals junctions. The van der Waals interface plays an important role in electronic and optoelectronic devices. Researchers are also trying to realize $2 \mathrm{D} / 3 \mathrm{D}$ heterojunction-based devices because the processing technology for 3D materials is well established. $\mathrm{ZnO}$ is generally recognized as a promising 3D material for short-wavelength optoelectronics devices due to its direct wide band gap of $3.37 \mathrm{eV}$ and a large exciton binding energy of $60 \mathrm{meV} \cdot{ }^{21-23}$ Thus, 
the heterostructure of phosphorene/ZnO can be potentially used for devices such as wide-band photodetectors.

Often, 2D heterostructures exhibit poor interfaces, especially when formed with a 3D material. The device fabrication process characteristically requires a thermal annealing process to improve this interface. However, improved thermal stability after 2D material deposition provides additional flexibility to deposit subsequent layers needed for a device. However, this annealing process is rarely investigated in the reported phosphorene/3D material-based devices. The selection of the annealing conditions is estimated to influence both the properties of phosphorene film and the ultimate device performance. Herein, we investigated the effect of annealing temperature on the Raman phonon peaks of a phosphorene/Aldoped $\mathrm{ZnO}$ (AZO) heterostructure having different types of passivation layers. We have also made heterostructure diodes and studied the effect of annealing temperature on the ideality factor to point out the utility of this passivation.

\section{Methods}

Bulk black phosphorus crystals were prepared using red phosphorus, $\mathrm{Sn}$, and $\mathrm{SnI}_{4}$ in the mass ratio $42: 2: 1$ following the method developed by Nilges. ${ }^{24}$ The materials were loaded into a $25 \mathrm{~cm}$ long fused silica ampoule. The ampoule was then evacuated to $10^{-5} \mathrm{~Pa}$ and sealed using an oxyacetylene torch. In the next step, the ampoule was loaded into a furnace, and temperature was slowly ramped to the growth condition, where the temperatures of the source and growth ends of the ampoule were kept at $650{ }^{\circ} \mathrm{C}$ and $600{ }^{\circ} \mathrm{C}$, respectively. This temperature condition was maintained for two hours and then ramped down, and the ampoule was allowed to cool down to room temperature. After the completion of cooling, the black phosphorus crystals were obtained by breaking the ampoule in a glove box. Few-layer black phosphorene flakes were deposited by mechanically exfoliating the bulk black phosphorus crystal onto AZO/Si at room temperature. A $20 \mathrm{~nm}$ AZO thin film was deposited on a Si substrate by atomic layer deposition (Savannah series from Cambridge Nano Tech Inc.). Prior to AZO deposition, the Si substrates were rinsed thoroughly with trichloroethylene, acetone, isopropanol, and deionized (DI) water and purged by $99.999 \%$ pure nitrogen gas to remove dust particles and other contaminants. Finally, these phosphorene/ $\mathrm{AZO} / \mathrm{Si}$ samples were passivated by $\mathrm{Al}_{2} \mathrm{O}_{3}, \mathrm{Si}_{3} \mathrm{~N}_{4}$, and $\mathrm{Al}_{2} \mathrm{O}_{3}$ / $\mathrm{Si}_{3} \mathrm{~N}_{4}$ stack deposited by plasma-enhanced atomic layer deposition (PE-ALD) (Fiji 200 Gen 2 from UltraTech). The thicknesses of the $\mathrm{Al}_{2} \mathrm{O}_{3}$ and $\mathrm{Si}_{3} \mathrm{~N}_{4}$ passivation layers were kept constant, $6.5 \mathrm{~nm}$ in all the samples. Annealing was carried out in RTP$600 \mathrm{~S}$ for 60 seconds under a flow of Ar at $10 \mathrm{sccm}$.

The fabrication process for a phosphorene/AZO p-n heterojunction diode is shown in Fig. 5(a). Initially, $60 \mathrm{~nm}$ of $\mathrm{SiO}_{2}$ was deposited on a cleaned Si substrate by high density plasma enhanced chemical vapor deposition (Apex SLR ICP). Then, a $20 \mathrm{~nm}$ AZO thin film was deposited on the sample by atomic layer deposition. Subsequently, phosphorene $(\sim 15 \mathrm{~nm})$ was deposited by mechanically exfoliating the bulk black phosphorus. Then, this sample was passivated with a $\mathrm{Si}_{3} \mathrm{~N}_{4}$ thin film
(6.5 $\mathrm{nm}$ ) deposited by PE-ALD. To reduce the pin-hole in the device, the $\mathrm{Al}_{2} \mathrm{O}_{3}$ film of $90 \mathrm{~nm}$ was deposited on top of the nitride. Then, a three-step direct write lithography process was used. In the first step, the alignment marks were patterned on the sample and then dry etched by an Oxford etcher (180-ICP). In the second step, two holes: one in $\mathrm{AZO}$ and the other in phosphorene, were made by dry etching the $\mathrm{Si}_{3} \mathrm{~N}_{4} / \mathrm{Al}_{2} \mathrm{O}_{3}$ stack. Next, the patterning of contact pads was carried out. Finally, $\mathrm{Ti}$ $(30 \mathrm{~nm}) / \mathrm{Au}(60 \mathrm{~nm})$ electrodes were deposited by metal physical vapor deposition and lift-off.

A micro-zone optical microscope with a CCD camera was used to place the few layer phosphorene on the AZO/Si substrate during the exfoliation process. The thickness and surface image of few layer phosphorene were obtained by a Bruker Nanoscope atomic force microscope (AFM) in the contact mode. The Raman spectra of these samples were obtained using confocal Raman spectroscopy (Witec Alpha300R) with an Ar laser at $514.5 \mathrm{~nm}$ as the excitation source. The Raman spectroscopy system had a CCD detector. To focus the laser beam and to obtain the Raman signal, a $50 \times$ objective lens was used. The spot size of laser beam was $0.5 \mu \mathrm{m}$. Identical conditions were employed to obtain the Raman spectra for all the samples. A Keithley source meter (2611A) was used to measure the currentvoltage characteristics of the fabricated phosphorene/AZO diodes.

\section{Results}

The thicknesses of the phosphorene flakes in heterostructure samples were determined by AFM. Fig. 1 shows the AFM height image and height profile of phosphorene flake passivated by the $\mathrm{Si}_{3} \mathrm{~N}_{4}$ layer. The height profiles confirm that all the samples have phosphorene flake thicknesses in the range of 8-16 nm.

Raman peaks are related to the in-plane $\left(\mathrm{A}_{\mathrm{g}}{ }^{2}\right.$ and $\left.\mathrm{B}_{2 \mathrm{~g}}\right)$ and out-of-plane $\left(\mathrm{A}_{\mathrm{g}}{ }^{1}\right)$ vibrational modes. Probe power was found to be an important parameter herein. Powers in excess of $23 \mathrm{~mW}$ $\mathrm{cm}^{-2}$ were found to damage phosphorene, leading to peak position shifts, even with the passivation layers. Power densities in the range from 2 to $5 \mathrm{~mW} \mathrm{~cm} \mathrm{~cm}^{-2}$ were used to avoid the damage. The effect of annealing temperature on the Raman spectra is shown in Fig. 2(a-c) for $\mathrm{Si}_{3} \mathrm{~N}_{4}, \mathrm{Al}_{2} \mathrm{O}_{3}$, and $\mathrm{Al}_{2} \mathrm{O}_{3} / \mathrm{Si}_{3} \mathrm{~N}_{4}$ passivation layers, respectively. Raman spectroscopy was performed on the same sample in the as-deposited state

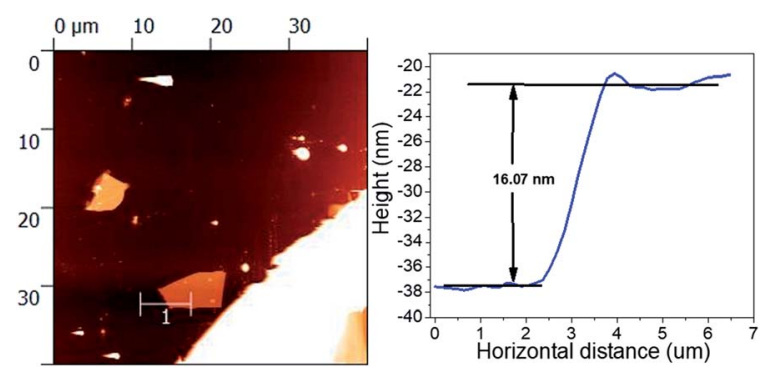

Fig. 1 AFM image and the corresponding AFM height profile of the typical phosphorene nanosheet. 

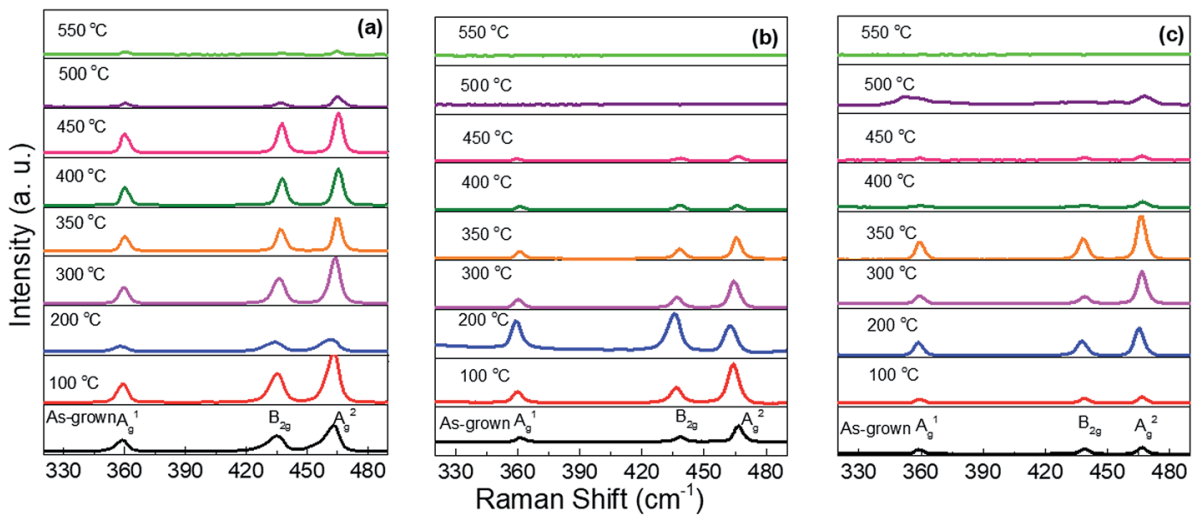

Fig. 2 Raman spectra of the phosphorene/AZO samples passivated by (a) $\mathrm{Si}_{3} \mathrm{~N}_{4}$, (b) $\mathrm{Al}_{2} \mathrm{O}_{3}$, and (c) $\mathrm{Si}_{3} \mathrm{~N}_{4} / \mathrm{Al}_{2} \mathrm{O}_{3}$ layers, annealed at different temperatures.

(un-annealed) as well as after each step in a series of increasing temperature anneals from $100{ }^{\circ} \mathrm{C}$ to $600{ }^{\circ} \mathrm{C}$. The sample with a $\mathrm{Si}_{3} \mathrm{~N}_{4}$ passivation layer demonstrated clear phonon peaks of in-plane $\left(\mathrm{A}_{\mathrm{g}}{ }^{2}\right.$ and $\left.\mathrm{B}_{2 \mathrm{~g}}\right)$ and out-of-plane $\left(\mathrm{A}_{\mathrm{g}}{ }^{1}\right)$ vibrational modes. Peak heights started to degrade at $450{ }^{\circ} \mathrm{C}$, with visible peaks up to $550{ }^{\circ} \mathrm{C}$ annealing. In comparison, samples passivated by $\mathrm{Al}_{2} \mathrm{O}_{3}$ show degradation at temperature as low as $300{ }^{\circ} \mathrm{C}$ and also exhibit loss of peaks at $450{ }^{\circ} \mathrm{C}$; samples with $\mathrm{Al}_{2} \mathrm{O}_{3} / \mathrm{Si}_{3} \mathrm{~N}_{4}$ hybrid layers have intermediate results with degradation beginning at $400{ }^{\circ} \mathrm{C}$ and peaks persisting up to a $500{ }^{\circ} \mathrm{C}$ anneal.

Shifts in the Raman peak position are typically attributed to the effect of stress. Blue shifts indicate increase in the compressive stress or decrease in the tensile stress. Phosphorene has a puckered honeycomb structure; thus, so biaxial strain can be present in both the zigzag and armchair directions. ${ }^{25}$ The peak positions are plotted in Fig. 3(a-c). To help evaluate this shift, the peak positions for a similar flake without a passivation layer are shown as a dashed line. The results obtained for the unannealed samples show the effect of the passivation layer stress on phosphorene; the out-of-plane mode shows that the effects of annealing is similar to that in the case of the in-plane peaks; the effects are less pronounced for in-plane peaks as can be expected.

The annealing results can be divided into three regimes: a low temperature red shift, a medium temperature blue shift, and high temperature degradation. The red shift occurs for anneals up to $200{ }^{\circ} \mathrm{C}$. It is especially pronounced for the $\mathrm{Si}_{3} \mathrm{~N}_{4}$ passivated sample. The blue shift occurs between 200 and $400{ }^{\circ} \mathrm{C}$ anneals. Peak positions slightly change between $400{ }^{\circ} \mathrm{C}$ and $450^{\circ} \mathrm{C}$. The behavior of the samples for high temperature
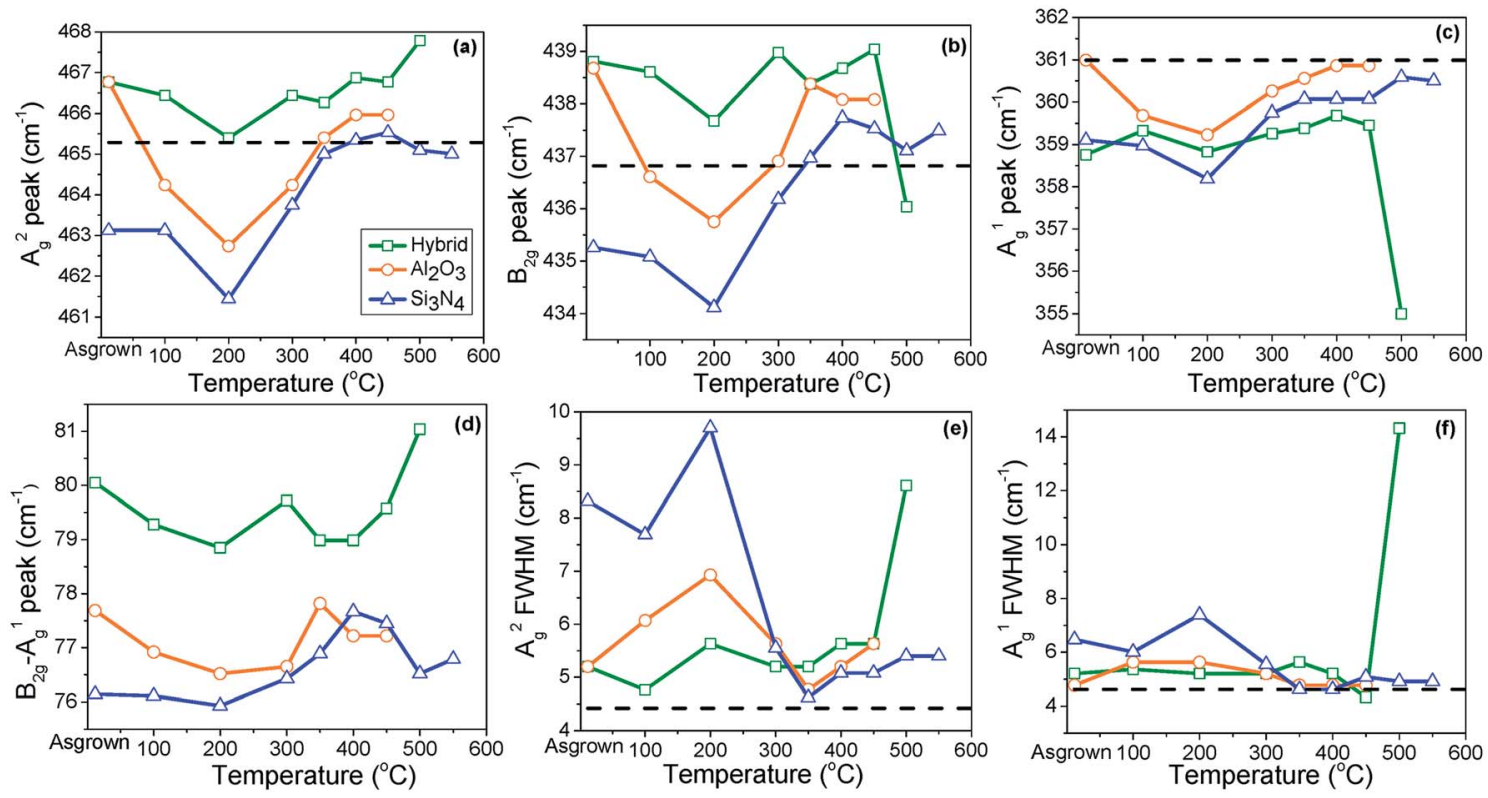

Fig. 3 Raman shifts of (a) $A_{g}{ }^{2}$, (b) $B_{2 g}$, and (c) $A_{g}{ }^{1}$ vibrational modes at different annealing temperatures. (d) The delta Raman shift between $B_{2 g}$ and $A_{g}{ }^{1}$ vibrational modes at different annealing temperatures. The full-width at half maximum (FWHM) variation of (e) $A_{g}{ }^{2}$ and (f) $A_{g}{ }^{1}$ phonon modes with annealing temperature for different samples. 
$\left(>400{ }^{\circ} \mathrm{C}\right.$ ) anneals was strongly passivation dependent. As abovementioned, $\mathrm{Al}_{2} \mathrm{O}_{3}$ and $\mathrm{Al}_{2} \mathrm{O}_{3} / \mathrm{Si}_{3} \mathrm{~N}_{4}$ samples did not show Raman peaks once annealed at 550 and $500{ }^{\circ} \mathrm{C}$, respectively, due to deterioration of phosphorene. Peak positions were erratic at the highest anneal temperatures for each sample. Presumably, this was due to the extensive crystal degradation. The energy spacing between the $\mathrm{A}_{\mathrm{g}}{ }^{1}$ and $\mathrm{B}_{2 \mathrm{~g}}$ modes decreases when the material is stretched, but increases under compression. ${ }^{25}$ In our study, the energy spacing between $\mathrm{A}_{\mathrm{g}}{ }^{2}$ and $\mathrm{B}_{2 \mathrm{~g}}$ modes returns to its low temperature value at higher annealing temperatures for the $\mathrm{Si}_{3} \mathrm{~N}_{4}$ passivated structure, as shown in Fig. 3(d). These observations differ from those reported in a previous study by Late, which indicated a linear red shift with the increasing temperatures up to $400{ }^{\circ} \mathrm{C}$ on a $\mathrm{SiO}_{2} / \mathrm{Si}$ substrate. ${ }^{26}$ This could be due to sample differences. In Late's report, no passivation layer was used on the phosphorene flakes; this allowed free expansion of phosphorene by annealing. ${ }^{26}$ The red shift in phosphorene is due to stretch in the zigzag direction. ${ }^{27}$ The energy spacing is higher for $\mathrm{Al}_{2} \mathrm{O}_{3}$ and $\mathrm{Si}_{3} \mathrm{~N}_{4} / \mathrm{Al}_{2} \mathrm{O}_{3}$ passivation layers as compared to that for the $\mathrm{Si}_{3} \mathrm{~N}_{4}$ passivation layer, as shown in Fig. 3(d). This indicates lower Raman peak intensities for $\mathrm{Al}_{2} \mathrm{O}_{3}$ and $\mathrm{Si}_{3} \mathrm{~N}_{4} / \mathrm{Al}_{2} \mathrm{O}_{3}$ passivation layers as compared to those for the $\mathrm{Si}_{3} \mathrm{~N}_{4}$ passivation layer. ${ }^{25}$

There are two possible reasons for the behavior observed in Fig. 3(a-d). The first is out-diffusion of $\mathrm{Zn}$ atoms from the AZO film. The atomic radius of the $\mathrm{Zn}$ atoms $(1.42 \AA)$ is larger than the radius of $\mathrm{P}$ atoms $(0.98 \AA)$. Thus, the incorporation of $\mathrm{Zn}$ in phosphorene can result in a lattice distortion, generating a compressive strain that compensates the already present tensile strain in phosphorene. ${ }^{22,26}$ Since diffusion coefficients increase exponentially with temperature, the amount of $\mathrm{Zn}$ incorporation into the phosphorene layer would increase sharply with annealing temperature. The second possible explanation is that the stress in the passivation layers is decreased by thermal annealing; this reduces the stress in phosphorene. To further study this, we deposited the passivation layers directly on $4^{\prime \prime}$ silicon wafers and used wafer bowing to determine the average film stress. We replicated the anneals applied to the phosphorene samples, and the stress after each anneal was measured. The results are shown in Fig. 4. Because the deposition was directly conducted on silicon rather than on the sample structure (phosphorene on AZO), the absolute values of the stress in these films may be different in this measurement. However, the annealing trends should show similar effects. Both films show a pronounced change in stress upon anneals of $300-450{ }^{\circ} \mathrm{C}$. This effect has been reported elsewhere. ${ }^{28}$ It is believed to be due to a densification caused by the loss of hydrogen from the films. This leads to tensile strain in the passivation layer when it is deposited on conventional materials. When it is deposited on a 2D material, the weak interplane forces will permit significant strain relaxation, but some degree of tensile strain will still exist. Thus, the densification of the passivation layer leads to compressive strain in phosphorene, producing the blue shift observed in Fig. 3.

It is tempting to ascribe the medium temperature shift in the Raman position (Fig. 2), which occurs from 200 to $400{ }^{\circ} \mathrm{C}$, with this $\mathrm{H}_{2}$ loss in the passivation layers, but there is an apparent difference in temperature. However, there are a few reasons to be skeptical of this apparent temperature difference. As described in the methods section, the samples were annealed in an optically heated rapid thermal processing system. The observed temperature in this system is the read out for a thermocouple in contact with a $4^{\prime \prime}$ silicon wafer. For the work summarized in Fig. 4, this is the actual coated wafer, whereas the work described in Fig. 3 was conducted on small samples $\left(\sim 1 \mathrm{~cm}^{2}\right)$ that were placed on top of the monitored $4^{\prime \prime}$ silicon wafer in the RTP system. Poor thermal contact could be responsible for some of this difference. More significant, however, is the fact that we are heating optically. Phosphorene has a short absorption length in the optical region, and the thin layer provides a very small thermal mass. ${ }^{29}$ Therefore, it is possible that the temperature of phosphorene and adjacent passivation layer significantly exceeds the measured temperature during heating transients due to the poor thermal contact of the system.

The crystalline properties of phosphorene were also investigated for different annealing temperatures by calculating FWHM of $\mathrm{A}_{\mathrm{g}}{ }^{2}$ and $\mathrm{A}_{\mathrm{g}}{ }^{1}$ phonon peaks, as shown in Fig. 3(e and f), respectively. The FWHM of phonon peaks is a good indicator of the crystalline quality. It is noteworthy to mention that the heights of peaks decrease abruptly between $100{ }^{\circ} \mathrm{C}$ and $200{ }^{\circ} \mathrm{C}$, as shown in Fig. 2(a). The highest decrement is observed for $200{ }^{\circ} \mathrm{C}$ annealing temperature. The FWHM values of these peaks are large for this temperature range (from as-grown to $200{ }^{\circ} \mathrm{C}$ ), as shown in Fig. 3(e and f). This indicates that the quality of the phosphorene flake is low in this temperature
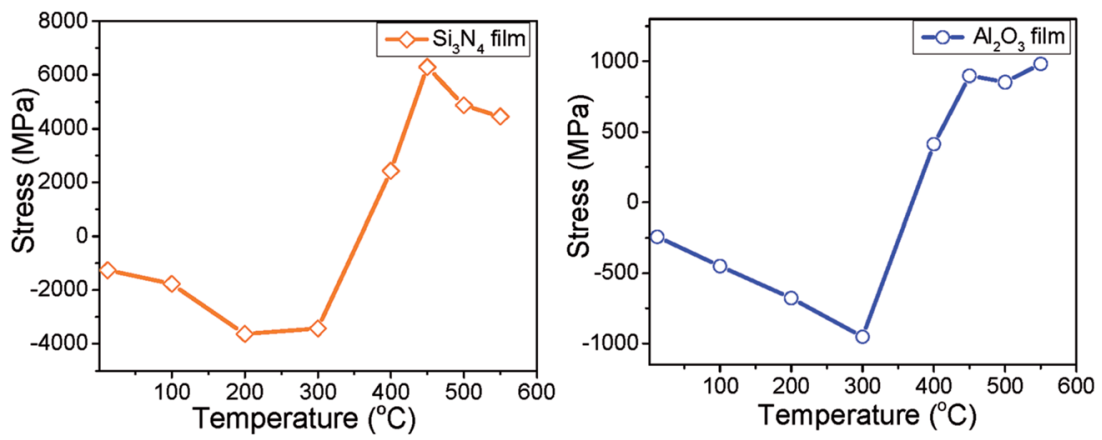

Fig. 4 Stress in the passivation layers as a function of annealing temperature. 
range; this results in the lower heights of peaks. It was observed that annealing at $350{ }^{\circ} \mathrm{C}$ produced phonon peaks with a much lower FWHM. Measurements of bulk black phosphorus indicated a FWHM of about $5 \mathrm{~cm}^{-1}$, comparable to the annealing results. Nitride-passivated films show marginally better results than the other samples. The decomposition of the $\mathrm{Al}_{2} \mathrm{O}_{3} / \mathrm{Si}_{3} \mathrm{~N}_{4}$ passivated structure at $500{ }^{\circ} \mathrm{C}$ is clearly visible in this plot. The return of the FWHM to the bulk value is an interesting result. If the strain relaxation was driven by the $\mathrm{Zn}$ up-diffusion, an increase in the in the peak width would be expected due to alloy broadening effects related to the disorder in the crystal. The fact that this was not seen suggested that this was not the case for our samples. The small FWHM in the high temperatureannealed $\mathrm{Si}_{3} \mathrm{~N}_{4}$-coated samples strongly suggests that the nitride acts as a diffusion barrier for phosphorus, allowing the crystalline layer to not only remain intact but actually improve its crystallinity for anneals that would otherwise destroy the material through sublimation. The upper limit for this effect is not known, but is likely to exceed the maximum temperature of $550{ }^{\circ} \mathrm{C}$ studied herein.

Finally, we have investigated that why the $\mathrm{Si}_{3} \mathrm{~N}_{4}$ passivation layer allows annealing at higher temperatures. Black phosphorus is known to be most stable of the various allotropes. However, it is also known to sublime at high temperatures. While the bulk material (black phosphorous) remains reasonably stable up to $550{ }^{\circ} \mathrm{C}$, phosphorene degrades at temperatures as low as $400{ }^{\circ} \mathrm{C}$, as noted by Liu. ${ }^{30}$ This is common for many nanostructures, including nanoparticles, nanotubes, and nanowires, and is believed to be due to the large surface to volume ratio. It is reasonable to suspect that the nitride layer acts as a diffusion barrier, blocking phosphorene decomposition by impeding the subliming flux of phosphorus atoms. Ito et al. studied the high temperature diffusion of phosphorus in thin nitride on top of silicon. ${ }^{31}$ For 30 minute anneals at $1000{ }^{\circ} \mathrm{C}$, the dose of phosphorus in the wafer is reduced by three orders of magnitude for a $3.4 \mathrm{~nm}$ nitride and nearly five orders of magnitude for a $9.2 \mathrm{~nm}$ nitride layer as compared to that of a $\mathrm{SiO}_{2}$ layer. Since the dose is proportional to the flux, it is clear that at this temperature, nitride is an effective diffusion barrier. Caution should be exercised while extrapolating from processes occurring at $1000{ }^{\circ} \mathrm{C}$ to processes occurring under the conditions used here, but note that reduction in the temperature will only reduce the diffusion coefficient further and nitride deposited is amorphous; thus, there will be no grain boundary diffusion effect at low temperatures.

Fig. 5 shows the schematic of the fabricated phosphorene/ AZO heterojunction $\mathrm{p}-\mathrm{n}$ diode. Fig. 5(b) illustrates the current-voltage $(I-V)$ characteristics of device measured at different annealing temperatures. To ensure that the diode represents the heterojunction and not a contact, resistors of both phosphorene and $\mathrm{AZO}$ with the same $\mathrm{Ti} / \mathrm{Au}$ contacts were tested as well. These show low resistance ohmic behavior, as shown in Fig. 5(c). It is well known that AZO has n-type conductivity; this indicates the p-type nature of phosphorene. The forward bias ideality factor was determined after annealing at different temperatures, as shown in Fig. 5(d). The series resistance at $1.2 \mathrm{~V}$ dropped slightly with annealing, from $24 \mathrm{k} \Omega$ to $15 \mathrm{k} \Omega$, suggesting an improvement in layer conductivity or a reduction in the contact resistance. The ideality factor decreased with an increase of annealing temperature; this
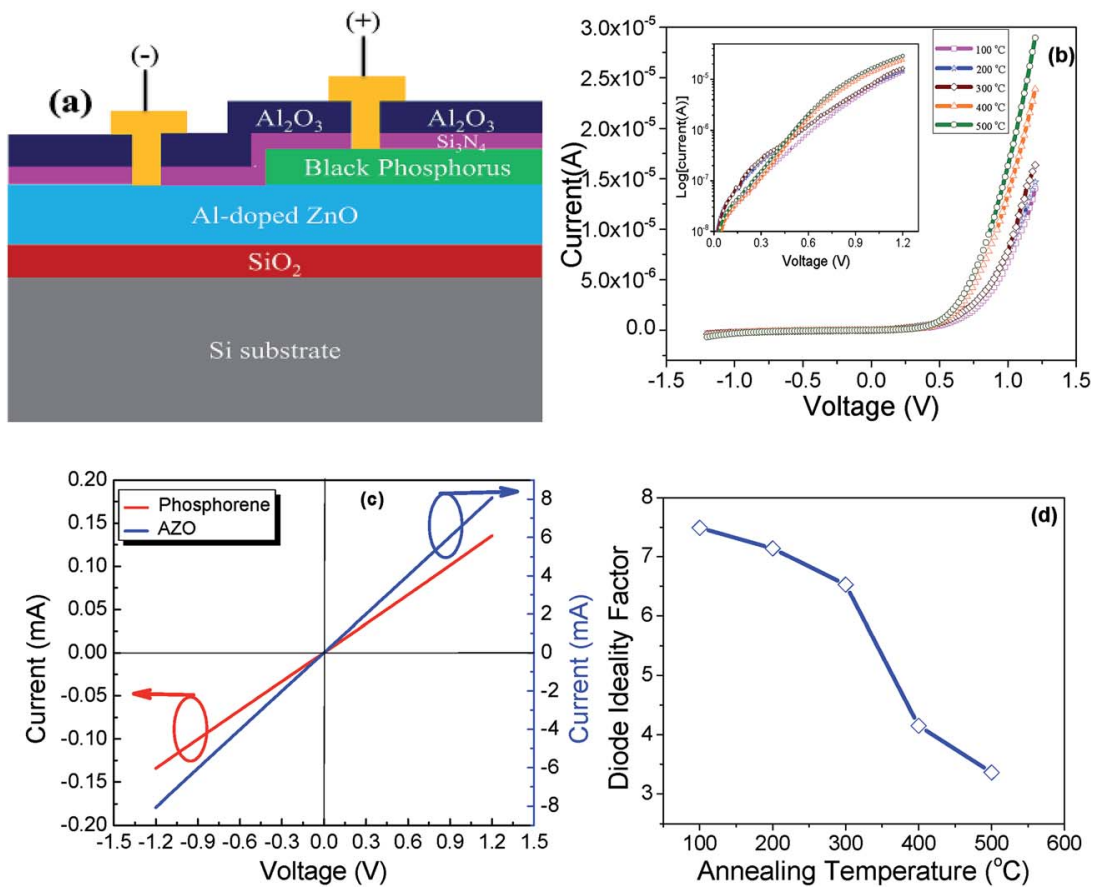

Fig. 5 (a) Device structure. (b) $I-V$ characteristics of the phosphorene/AZO heterojunction diode at different annealing temperatures. Inset shows the semilog scale. (c) I-V characteristics showing ohmic behavior by the Ti/Au contact on phosphorene and AZO layers. (d) Ideality factor versus annealing temperature. 
indicated improved quality, achieving a minimum value of 3.3 after annealing at $500{ }^{\circ} \mathrm{C}$ (Fig. 5(d)). Rectifying $I-V$ characteristics disappear and ohmic behavior is observed after annealing at $550{ }^{\circ} \mathrm{C}$.

Annealing can have multiple effects including change in the conductivity of the films by changing the carrier concentration or mobility, change in the specific resistance of the contacts, and change in the defect density in the bulk or at the interface. ${ }^{\mathbf{1 9}}$ The series resistance of the device can be inferred from the slope of the $I-V$ characteristics at large forward bias. Herein, we see only a modest difference and thus rule out the first two possible effects. We, therefore, believe that annealing reduces the defect concentration and therefore the recombination current. This can be seen most easily in the small-value $(100 \mathrm{mV}$ to $300 \mathrm{mV}$ ) of forward bias characteristics shown in the inset of Fig. 5(b). A reduction of the current in this region, along with a drop in the ideality factor, is a clear indication that annealing is reducing the recombination rate. At higher bias values, carriers are injected from the n-region to the $\mathrm{p}$-region and vice versa. Therefore, the current is due to the diffusion of carriers in the quasi-neutral regions. The diffusion coefficient improves after higher temperature annealing due to the reduction of the defect concentration. Perhaps more importantly, however, the forward current is proportional to $\exp \left(V_{\mathrm{A}} / n k T\right)$. As the ideality factor $(n)$ decreases, the value of the exponent increases for a given applied voltage, and thus, the current increases exponentially. Raman measurements showed that the phosphorene crystalline quality was improved for anneals at higher temperature; this resulted in a reduction of the defect concentration. We cannot tell from this data if this improvement is at the interface or in the bulk. The loss of rectifying behavior after annealing at $550{ }^{\circ} \mathrm{C}$ corresponds to the reduction in the Raman peak intensities and the increase in the peak width for the nitride-passivated samples at this temperature. All these observations suggest a sharp drop in the crystal quality.

\section{Conclusions}

Herein, three samples having a few-layer phosphorene/AZO heterostructure were passivated by $\mathrm{Al}_{2} \mathrm{O}_{3}, \mathrm{Si}_{3} \mathrm{~N}_{4}$, and $\mathrm{Al}_{2} \mathrm{O}_{3}$ / $\mathrm{Si}_{3} \mathrm{~N}_{4}$ layers separately using PE-ALD. These phosphorene/AZO heterostructures were annealed at different temperatures, and the thermal stability was measured by Raman spectroscopy. AFM measurements confirmed that all samples had phosphorene flakes with thicknesses in the range of 8-16 nm. The $\mathrm{Si}_{3} \mathrm{~N}_{4}$-passivated structure demonstrated highest thermal sustainability, showing clear vibrational modes for annealing temperature in the range of $100-550{ }^{\circ} \mathrm{C}$. The phonon peaks red shifted after annealing up to $200{ }^{\circ} \mathrm{C}$; this suggested the generation of tensile strain and/or reduction of compressive strain. For annealing temperatures above $200{ }^{\circ} \mathrm{C}$, phosphorene produced a blue shift in the in-plane $\left(\mathrm{B}_{2 \mathrm{~g}}\right.$ and $\left.\mathrm{Ag}_{\mathrm{g}}{ }^{2}\right)$ and out-ofplane $\left(\mathrm{A}_{\mathrm{g}}{ }^{1}\right)$ vibrational modes, returning to the peak positions for the unannealed sample. Thus, strain relaxation took place at higher annealing temperature for the $\mathrm{Si}_{3} \mathrm{~N}_{4}$ structure. We believe that the effect of annealing on the strain of the passivation layers plays a critical role in these shifts. The fabricated
phosphorene/AZO heterojunction diode shows improvement in the quality of diode at higher annealing temperatures. Herein, we present a study that has the potential to accelerate further developments in the field of high-performance phosphorene/ZnO heterojunction-based wide-band devices such as photodetectors.

\section{Conflicts of interest}

There are no conflicts to declare.

\section{Acknowledgements}

Part of this work was carried out at the Nano Center of University of Minnesota, which was supported by the National Science Foundation through NNCI (Award ECCS-1542202). SKP and NI also received support through this award. We are also grateful to the atomic force microscopy (AFM) and Raman spectroscopy facilities equipped at the Characterization Facility at the University of Minnesota.

\section{References}

1 K. Kim, J. Y. Choi, T. Kim, S. H. Cho and H. J. Chung, Nature, 2011, 479, 338-344.

2 Q. H. Wang, K. K. Zadeh, A. Kis, J. N. Coleman and M. S. Strano, Nat. Nanotechnol., 2012, 7, 699-712.

3 F. H. L. Koppens, T. Mueller, P. Avouris, A. C. Ferrari, M. S. Vitiello and M. Polini, Nat. Nanotechnol., 2014, 9, 780-793.

4 F. Schwierz, Nat. Nanotechnol., 2010, 5, 487-496.

5 G. Fiori, F. Bonaccorso, G. Iannaccone, T. Palacios, D. Neumaier, A. Seabaugh, S. K. Banerjee and L. Colombo, Nat. Nanotechnol., 2014, 9, 768-779.

6 K. S. Novoselov, V. I. Fal'ko, L. Colombo, P. R. Gellert, M. G. Schwab and K. Kim, Nature, 2012, 490, 192-200.

7 P. Avouris, Z. Chen and V. Perebeinos, Nat. Nanotechnol., 2007, 2, 605-615.

8 M. K. Jana, A. Singh, D. J. Late, C. R. Rajamathi, K. Biswas, C. Felser, U. V. Waghmare and C. N. R. Rao, J. Phys.: Condens. Matter, 2015, 27, 285401.

9 D. J. Late, S. N. Shirodkar, U. V. Waghmare, V. P. Dravid and C. N. Rao, ChemPhysChem, 2014, 15, 1592-1598.

10 L. Li, Y. Yu, G. J. Ye, Q. Ge, X. Ou, H. Wu, D. Feng, X. H. Chen and Y. Zhang, Nat. Nanotechnol., 2014, 9, 372-377.

11 Y. Cai, G. Zhang and Y. W. Zhang, Sci. Rep., 2014, 4, 66776683.

12 H. Liu, A. T. Neal, Z. Zhu, Z. Luo, X. Xu, D. Tomanek and P. D. Ye, ACS Nano, 2014, 8, 4033-4041.

13 H. Liu, Y. Du, Y. Deng and P. D. Ye, Chem. Soc. Rev., 2015, 44, 2732-2743.

14 J. Dai and X. Zeng, J. Phys. Chem. Lett., 2014, 5, 1289-1293. 15 F. Xia, H. Wang, D. Xiao, M. Dubey and A. Ramasubramaniam, Nat. Photonics, 2014, 8, 899-907.

$16 \mathrm{~K}$. W. Ang, Z. P. Ling and J. Zhu, IEEE International Conference on Digital Signal Processing, 2015, p. 1223. 
17 P. K. Kannan, D. J. Late, H. Morgan and C. S. Rout, Nanoscale, 2015, 7, 13293-13312.

18 P. J. Jeon, Y. T. Lee, J. Y. Lim, J. S. Kim, D. K. Hwang and S. Im, Nano Lett., 2016, 16, 1293-1298.

19 Y. Deng, Z. Luo, N. J. Conrad, H. Liu, Y. Gong, S. Najmaei, P. M. Ajayan, J. Lou, X. Xu and P. D. Ye, ACS Nano, 2014, 8, 8292-8299.

20 P. Gehring, R. Urcuyo, D. L. Duong, M. Burghard and K. Kern, Appl. Phys. Lett., 2015, 106, 233110.

21 S. K. Pandey, S. K. Pandey, C. Mukherjee, P. Mishra, M. Gupta, S. R. Barman, S. W. D'Souza and S. Mukherjee, J. Mater. Sci.: Mater. Electron., 2013, 24, 2541-2547.

22 S. K. Pandey, S. K. Pandey, U. P. Deshpande, V. Awathi, A. Kumar, M. Gupta and S. Mukherjee, Semicond. Sci. Technol., 2013, 28, 085014.

23 S. J. Pearton, D. P. Norton, K. Ip, Y. W. Heo and T. Steiner, J. Vac. Sci. Technol., B: Microelectron. Nanometer Struct.Process., Meas., Phenom., 2004, 22, 932-948.
24 M. Köpf, N. Eckstein, D. Pfister, C. Grotz, I. Krüger, M. Greiwe, T. Hansen, H. Kohlmann and T. Nilges, J. Cryst. Growth, 2014, 405, 6-17.

25 R. Fei and L. Yang, Appl. Phys. Lett., 2014, 105, 083120.

26 D. J. Late, ACS Appl. Mater. Interfaces, 2015, 7, 5857-5862.

27 Z. P. Ling and K. W. Ang, APL Mater., 2015, 3, 126104.

28 A. Bagolini, A. S. Savoia, A. Picciotto, M. Boscardin, P. Bellutti, N. Lamberti and G. Caliano, J. Micromech. Microeng., 2015, 25, 015012.

29 S. Zhang, J. Yang, R. Xu, F. Wang, W. Li, M. Ghufran, Y. W. Zhang, Z. Yu, G. Zhang, Q. Qin and Y. Lu, ACS Nano, 2014, 8, 9590-9596.

30 X. Liu, J. D. Wood, K. S. Chen, E. K. Cho and M. C. Hersam, J. Phys. Chem. Lett., 2015, 6, 773-778.

31 T. Ito, S. Hijiya, T. Nozaki, H. Arakawa, M. Shinoda and Y. Fukukawa, J. Electrochem. Soc., 1978, 125, 448-452. 